\title{
Dynamics of One-dimensional Self-gravitating Systems Using Hermite-Legendre Polynomials
}

\author{
Eric I. Barnes ${ }^{1}$, Robert J. Ragan \\ Department of Physics, University of Wisconsin - La Crosse
}

\begin{abstract}
The current paradigm for understanding galaxy formation in the universe depends on the existence of self-gravitating collisionless dark matter. Modeling such dark matter systems has been a major focus of astrophysicists, with much of that effort directed at computational techniques. Not surprisingly, a comprehensive understanding of the evolution of these self-gravitating systems still eludes us, since it involves the collective nonlinear dynamics of many-particle systems interacting via long-range forces described by the Vlasov equation. As a step towards developing a clearer picture of collisionless self-gravitating relaxation, we analyze the linearized dynamics of isolated one-dimensional systems near thermal equilibrium by expanding their phase space distribution functions $f(x, v)$ in terms of Hermite functions in the velocity variable, and Legendre functions involving the position variable. This approach produces a picture of phase-space evolution in terms of expansion coefficients, rather than spatial and velocity variables. We obtain equations of motion for the expansion coefficients for both test-particle distributions and self-gravitating linear perturbations of thermal equilibrium. This development presents the opportunity to avoid time-consuming $N$-body simulations that are limited by statistical uncertainty and provides a powerful analysis tool for understanding the relaxation to equilibrium.
\end{abstract}

\section{Introduction}

Over the past several decades, much evidence has been compiled supporting the idea that the baryonic mass visible in galaxies (stars, gas, and dust) comprises a small fraction of the total gravitating mass of such a system. The earliest evidence comes from observations of galactic motions within larger galaxy cluster systems. Individual galaxies had velocities that were too large to remain bound to the cluster, given the inferred amount of stellar mass (Zwicky 1937). However, the uncertainties associated with this analysis were large, and it took several more decades for more conclusive evidence to emerge. The rotation curves (circular speed versus galactocentric distance) of spiral galaxies are considered to be one

\footnotetext{
${ }^{1}$ EB gratefully acknowledges support from the Wisconsin Space Grant Consortium Research Infrastructre Program.
} 
of the clearest pieces of evidence for what has become known as dark matter surrounding galaxies. In general, these curves show circular speeds of stars and gas in spiral galaxies following solid-body-like rotation near their centers, then reaching a nearly constant value (Rubin \& Ford 1970). This contrasts with predictions based on the observed stellar/gas mass distributions in these galaxies, where the circular speed should peak and then decrease in the outer regions of a galaxy. Further studies of stellar kinematics in elliptical galaxies can hint at the need for dark matter, but the dynamics of such systems are more complex than for spiral galaxies, and interpretations are not as clear (Romanowsky et al. 2003).

In parallel with these inferences from galaxy dynamics, the idea of dark matter has also been supported by cosmological investigations. Numerical simulations of large-scale structure formation in the universe can reproduce the observed filamentary structure of galaxy clusters if dark matter is included (Navarro, Frenk, White 1996; Springel et al. 2005). Observations of the cosmic microwave background reveal features that can be described best when roughly $25 \%$ of the mass in the universe is dark matter (Spergel et al. 2003). A third route of evidence for dark matter around galaxies involves observations of gravitational lensing. Locations and magnifications of images of distant galaxies and quasars that form when their light is bent around intervening galaxies (or clusters of galaxies) indicate that the lensing galaxies should have masses larger than what can be accounted for from their visible components (Clowe et al. 2006; Williams \& Saha 2011).

The current paradigm assumes that dark matter must act collisionlessly. The argument supporting this assumption is as follows. Observations indicating the presence of dark matter have not shown indications of an edge to the dark matter halo. For example, there are no isolated spiral galaxy rotation curves where the circular speed of gas begins to show a Keplerian decrease at some distance. As a result, it is assumed that the dark matter structures around galaxies have much larger spatial extent than the visible components. The baryons that will eventually form stars (mostly Hydrogen gas) are initially mixed with the dark matter over these larger volumes, but the baryons will self-interact via forces other than gravity. This gives the baryons a cooling mechanism that is unavailable to dissipationless dark matter and allows gas to radiate energy away and sink towards the center of the dark matter structure (typically referred to as a halo). Further, collisional effects would lead to halos with more spherical shapes that observations of galaxy clusters would allow (Mohr et al. 1995).

The previously mentioned cosmological simulations of structure formation have done more than simply suggest the reality of dark matter, they have also predicted its behavior on the scale of galaxies. It is generally agreed upon in the simulation community that dark matter halo mass density profiles have central cusps $\rho \propto r^{-\gamma}$ where $\gamma \approx 1$. The logarithmic density profiles then monotonically steepen as one moves away from the center (e.g. Navarro, Frenk, White 1997; Navarro et al. 2004). The consistency of the density behavior across mass scales, initial conditions, and simulation methods suggests that some simple underlying physics is at play in these self-gravitating collisionless systems. Further investigations into the kinematics of dark matter systems have likewise heightened the suggestion of a fundamental 
physical process driving the formation of mechanical equilibrium dark matter halos (Taylor \& Navarro 2001; Hansen \& Moore 2006; Lithwick \& Dalal 2011). Investigations of these threedimensional (3-d) systems involve a wide range of modes of evolution that contribute to the relaxation from initial conditions to a final equilibrium state. The radial orbit instability (Merritt \& Aguilar 1985), along with evaporation and ejection (Binney \& Tremaine 1987, Chapter 7), are examples of these modes.

In this paper we will consider a one-dimensional (1-d) self-gravitating collisionless system (which can also be formulated as a "sheet" model (Camm 1950). Compared to 3-d models, the 1-d model is easier to analyze while possessing the essential features of 3-d systems attractive long range forces and collisonless collective dynamics. However, it lacks some of the features of 3 -d systems like angular momentum and tidal forces. Though the model is formulated in terms of continuous distribution functions, it can also be considered as the $N \rightarrow \infty$ limit of system of $N$ particles with masses $m$, interacting via two-body gravitational attraction. The evolution of the phase-space distribution function is described by the the Vlasov equation (or collisionless Boltzmann equation),

$$
\frac{\mathrm{d} f}{\mathrm{~d} t}=\frac{\partial f}{\partial t}+v \frac{\partial f}{\partial x}+a(x) \frac{\partial f}{\partial v}=0
$$

where $f(x, v ; t)$ is the normalized distribution function

$$
\int_{-\infty}^{\infty} \int_{-\infty}^{\infty} f(x, v ; t) \mathrm{d} x \mathrm{~d} v=1 .
$$

The $t$ argument is implied in what follows. The density is obtained simply by integrating over velocities

$$
\lambda(x)=M \int_{-\infty}^{\infty} f(x, v) \mathrm{d} v
$$

where $M$ is a mass scale for the system (mass per unit area in the sheet model; the total mass $N m$ for particles). The acceleration $a(x)$ for 1 -d systems is calculated by simply taking the difference of the total masses on each side of $x$,

$$
\begin{aligned}
a(x) & =-g \int_{-\infty}^{x} \lambda(s) \mathrm{d} s+g \int_{x}^{\infty} \lambda(s) \mathrm{d} s \\
& =g\left(M_{>}-M_{<}\right),
\end{aligned}
$$

where $g$ is the 1-d analogue of Newton's gravitational constant, and $M_{>}$and $M_{<}$represent the mass to the right and left of any location $x$, respectively. Note the long-range nature of the interaction, which couples particles through the distance between them. Likewise, the density is non-local in phase space, in that it involves an integral over velocities.

Studies of such 1-d systems have a long history (Camm 1950). In general, much of the work can be categorized by dealing with either cosmological conditions (e.g. Valgeas 2006) where an additional non-self-gravitating potential energy term is included in the Hamiltonian (or periodic boundary conditions are used), or isolated systems where self-gravity is the only source of potential (e.g. Reidl \& Miller 1988; Koyama \& Konishi 2001; Schulz et al. 
2013). Within each of these categories, a variety of initial conditions have been investigated. Broadly speaking, initial conditions are typically near-equilibrium (e.g. Reidl \& Miller 1987) or far-from-equilibrium (e.g. Joyce \& Worrakitpoonpon 2011). The situations we investigate are isolated systems near thermal equilibrium. Such non-equilibrium systems might be considered to be in the final stages of condensation from uniform cosmological conditions, or perhaps in the aftermath of a collision in which two systems coalesce/pass through one another. We note that the absence of tidal forces in 1-d guarantees that non-overlapping systems can be considered as isolated, so that our discussion also applies to clusters of non-overlapping systems between encounters.

What follows here is a discussion of a method for finding solutions to a linearized version of Equation 1. Our approach is to expand the distribution function in terms of orthogonal functions. This method has been used previously, with Hermite polynomials to describe the velocity aspect of distributions (Reidl \& Miller 1988), and Fourier expansions for the position for cosmological models with periodic boundary conditions (Alvord \& Miller 2009; Reidl \& Miller 1987). The form of the thermal equilibrium distribution function for isolated systems very naturally suggests the use of Hermite polynomials for the velocity and Legendre polynomials in $\tanh (x)$ for the position.

The resulting linear set of equations of motion link the expansion coefficients $c_{m, n}(t)$. In this notation, $m$ and $n$ are the orders of the Hermite and Legendre polynomials, respectively. There are few couplings between the coefficients - in fact, the couplings are local, in that they are only between neighbors on the $(m, n)$ grid. This is rather fortuitous in light of the long-range nature of the forces, and gives a simple local continuity-type evolution of coefficients on the $(m, n)$ grid. Furthermore, the method provides a more efficient route to following the phase-space evolution of modestly perturbed systems, compared to $N$-body simulations.

\section{Thermal Equilibrium}

Based on the structure of Equation 1, any function of the single particle energy,

$$
\epsilon=\frac{1}{2} m v^{2}+m \phi(x)
$$

is a time-independent solution. Thermal equilibrium is a special case which case the distribution function has the separable Boltzmann form

$$
f_{0}(\epsilon)=A e^{-\beta \epsilon}=A e^{-\frac{\beta m v^{2}}{2}} e^{-\beta m \phi},
$$

where $\beta \equiv 1 / k_{B} T=1 /\left\langle m v^{2}\right\rangle$ is an energy scale (commonly referred to as the inverse temperature), and $A$ is a normalization constant. Upon substitution of Equations 4-5 into Equation 1, it is straightforward to obtain the thermal equilibrium distribution function, which is commonly written as,

$$
f_{0}(x, v)=A \operatorname{sech}^{2}\left(\frac{\beta g m M}{2} x\right) e^{-\frac{\beta m v^{2}}{2}}
$$


where $A=(g M / 4) \sqrt{\beta^{3} m^{3} / 2 \pi}$. The corresponding potential is,

$$
\begin{aligned}
\phi_{0}(x) & =\int_{-\infty}^{\infty} g|x-s| \lambda(s) \mathrm{d} s \\
& =\frac{2}{\beta m} \ln \left(2 \cosh \frac{\beta g m M}{2} x\right),
\end{aligned}
$$

from which we obtain the acceleration

$$
a_{0}(x)=-\frac{\partial \phi_{0}(x)}{\partial x}=-g M \tanh \frac{\beta g m M}{2} x .
$$

In terms of the quantities defined, the kinetic energy of the equilibrium state is

$$
K_{0}=M \int_{-\infty}^{\infty} \frac{v^{2}}{2} f_{0}(x, v) \mathrm{d} x \mathrm{~d} v=\frac{N}{2 \beta} .
$$

The equilibrium potential energy is likewise given by

$$
U_{0}=\frac{1}{2} \int_{-\infty}^{\infty} \lambda_{0}(x) \phi_{0}(x, v) \mathrm{d} x \mathrm{~d} v=\frac{N}{\beta}=2 K_{0},
$$

as required by the virial theorem for one dimension.

The Boltzmann nature of the one-dimensional self-gravitating system is a vital difference from the three-dimensional case. Mechanical equilibria of realistic three-dimensional selfgravitating systems always contain gradients in the kinetic temperature, $T_{\mathrm{K}} \propto\left\langle v^{2}\right\rangle$, that act as pressure support against gravity. Only the infinite mass and energy isothermal sphere has a constant temperature. This one-dimensional distribution function is a true thermal equilibrium, as the kinetic temperature is uniform throughout the equilibrium system.

For simplicity, we transform to dimensionless coordinates using the definitions,

$$
\chi=\frac{\beta g m M}{2} x \text { and } \varpi=\sqrt{\frac{\beta m}{2}} v .
$$

The scaled equilibrium distribution function is,

$$
\tilde{f}_{0}(\chi, \varpi)=\frac{2}{\beta g m M} \sqrt{\frac{2}{\beta m}} f_{0}=\frac{1}{2 \sqrt{\pi}} \operatorname{sech}^{2} \chi e^{-\varpi^{2}} .
$$

where tildes are used to indicate dimensionless functions, when a distinction is necessary. The Vlasov equation transforms to,

$$
\frac{\partial \tilde{f}}{\partial \tau}+\varpi \frac{\partial \tilde{f}}{\partial \chi}+\alpha(\chi) \frac{\partial \tilde{f}}{\partial \varpi}=0,
$$

where $\tau=\sqrt{\beta m / 2} g M t$ is the dimensionless time and $\alpha(\chi)=a /(g M)$ is the dimensionless acceleration function. 


\section{Orthogonal Polynomials}

The form of the equilibrium distribution function suggests a set of orthogonal functions to use as a basis for a polynomial expansion. We consider the expansion,

$$
\tilde{f}(\chi, \varpi)=\sum_{i, j} c_{i, j} G_{i j}(\chi, \varpi) \tilde{f}_{0}(\chi, \varpi),
$$

where the $c_{i, j}$ are real expansion coefficients. The $G_{i j}$ are functions defined by

$$
G_{i j}(\chi, \varpi)=\sqrt{\frac{2 j+1}{2^{i} i !}} H_{i}(\varpi) P_{j}(\tanh \chi)
$$

where the $H_{i}$ are Hermite polynomials of order $i$, and the $P_{j}$ are Legendre polynomials of order $j$. The $G_{i j}$ are constructed to be orthonormal to $f_{0}$, which serves as a weighting function,

$$
\int_{-\infty}^{\infty} \int_{-\infty}^{\infty} G_{i j}(\chi, \varpi) G_{i^{\prime} j^{\prime}}(\chi, \varpi)(\varpi) \tilde{f}_{0}(\chi, \varpi) \mathrm{d} \chi \mathrm{d} \varpi=\delta_{i i^{\prime}} \delta_{j j^{\prime}}
$$

We routinely use the Hermite polynomial orthogonality condition,

$$
\int_{-\infty}^{\infty} H_{i}(\varpi) H_{i^{\prime}}(\varpi) e^{-\varpi^{2}} \mathrm{~d} \varpi=\delta_{i i^{\prime}} 2^{i} \sqrt{\pi} i !
$$

where $\delta$ is the Kronecker delta. For the Legendre orthogonality condition, we can eliminate the factor $\operatorname{sech}^{2} \chi$ with the change of variables $u=\tanh \chi$ and $\mathrm{d} u=\operatorname{sech}^{2} \chi \mathrm{d} \chi$,

$$
\begin{aligned}
\int_{-\infty}^{\infty} P_{j}(\tanh \chi) P_{j^{\prime}}(\tanh \chi) \operatorname{sech}^{2} \chi \mathrm{d} \chi & = \\
\int_{-1}^{1} P_{j}(u) P_{j^{\prime}}(u) \mathrm{d} u & =\delta_{j j} \frac{2}{2 j+1} .
\end{aligned}
$$

Note that this substitution also maps infinite limits on any $\chi$ integral to the interval $[-1,1]$.

At thermal equilibrium, only the $i=0, j=0$ coefficient is nonzero. For an arbitrary distribution function $\tilde{f}(\chi, \varpi)$ perturbed from thermal equilibrium, the coefficients can be determined from

$$
c_{i, j}=\int_{-\infty}^{\infty} \int_{-\infty}^{\infty} G_{i, j}(\chi, \varpi) \tilde{f}(\chi, \varpi) \mathrm{d} \chi \mathrm{d} \varpi .
$$

This equation represents a transformation from phase space to a discrete $(i, j)$ grid of coefficients.

The expansion dictates that all mass must derive from the $(0,0)$ term,

$$
\begin{aligned}
\tilde{M}_{i, j} \equiv \frac{M_{i, j}}{M} & =\int_{-\infty}^{\infty} \int_{-\infty}^{\infty} \tilde{f}_{i, j} \mathrm{~d} \chi \mathrm{d} \varpi \\
& =\int_{-\infty}^{\infty} \int_{-\infty}^{\infty} c_{i, j} G_{i j}(\chi, \varpi) \tilde{f}_{0}(\chi, \varpi) \mathrm{d} \chi \mathrm{d} \varpi \\
& =c_{i, j} \delta_{i 0} \delta_{j 0},
\end{aligned}
$$


from which we obtain $\tilde{M}_{0,0}=c_{0,0}=1$. In a similar fashion, one can see that mass density $\tilde{\lambda}(\chi)$ derives only from $i=0$ terms,

$$
\begin{aligned}
\tilde{\lambda}(\chi) & =\int_{-\infty}^{\infty} \tilde{f}_{i, j} \mathrm{~d} \varpi \\
& =\int_{-\infty}^{\infty} \sum_{i, j} c_{i, j} G_{i j}(\chi, \varpi) \tilde{f}_{0}(\chi, \varpi) \mathrm{d} \varpi \\
& =\sum_{j} c_{0, j} \sqrt{2 j+1} P_{j}(\tanh \chi) \tilde{\lambda}_{0}(\chi) .
\end{aligned}
$$

\section{Linear Perturbations}

We now use the orthogonal polynomials developed in the previous section as a basis to study the dynamics of perturbations from thermal equilibrium. We consider distribution functions of the form,

$$
\tilde{f}=\tilde{f}_{0}+\delta \tilde{f}_{1}
$$

where $\tilde{f}_{1}$ is the perturbing function and $\delta \ll 1$ is an expansion parameter.

Using this perturbed $\tilde{f}$ in Equation 1 produces a modified Vlasov equation for the perturbing function (in terms of the previously defined dimensionless quantities),

$$
\frac{\partial \tilde{f}_{1}}{\partial \tau}+\varpi \frac{\partial \tilde{f}_{1}}{\partial \chi}+\alpha_{0}(\chi) \frac{\partial \tilde{f}_{1}}{\partial \varpi}=2 \varpi \alpha_{1}(\chi) \tilde{f}_{0}
$$

where we have used $\partial \tilde{f}_{0} / \partial \varpi=-2 \varpi \tilde{f}_{0}$. The accelerations are given by

$$
\begin{gathered}
\alpha_{0}(\chi)=-\int_{-\infty}^{\chi} \tilde{\lambda}_{0}\left(\chi^{\prime}\right) \mathrm{d} \chi^{\prime}+\int_{\chi}^{\infty} \tilde{\lambda}_{0}\left(\chi^{\prime}\right) \mathrm{d} \chi^{\prime}=-\tanh \chi \\
\alpha_{1}(\chi)=-\int_{-\infty}^{\chi} \tilde{\lambda}_{1}\left(\chi^{\prime}\right) \mathrm{d} \chi^{\prime}+\int_{\chi}^{\infty} \tilde{\lambda}_{1}\left(\chi^{\prime}\right) \mathrm{d} \chi^{\prime} .
\end{gathered}
$$

The term $2 \varpi \alpha_{1}(\chi) \tilde{f}_{0}$ is required by Newton's Third Law. In this equation, it has been written on the right hand side to signify that it is neither a convective nor an advective term. In fact, the right hand side is best characterized as a collision term as it represents the deflection of particles into and out of equilibrium due to the perturbation. Here, we ignore the second-order term describing the self-interaction of the perturbing particles, $\alpha_{1} \partial \tilde{f}_{1} / \partial \varpi$.

We now express the perturbing distribution function in terms of the Hermite and Legendre polynomials discussed earlier,

$$
\tilde{f}_{1}=\sum_{i, j} c_{i, j} \sqrt{\frac{2 j+1}{2^{i} i !}} H_{i}(\varpi) P_{j}(\tanh \chi) \frac{\operatorname{sech}^{2} \chi e^{-\varpi^{2}}}{2 \sqrt{\pi}},
$$

where $c_{0,0}=0$ since the equilibrium contribution has already been removed. This guarantees that the perturbations are massless. Using Equation 19 in Equation 22, the perturbing 
acceleration is found to be,

$$
\begin{aligned}
\alpha_{1}(\chi)= & \sum_{j} c_{0, j} \sqrt{2 j+1}\left[\int_{\chi}^{\infty} P_{j}\left(\tanh \chi^{\prime}\right) \frac{\operatorname{sech}^{2}}{2} \chi^{\prime} \mathrm{d} \chi^{\prime}-\right. \\
& \left.\int_{-\infty}^{\chi} P_{j}\left(\tanh \chi^{\prime}\right) \frac{\operatorname{sech}^{2}}{2} \chi^{\prime} \mathrm{d} \chi^{\prime}\right] .
\end{aligned}
$$

Upon making the substitution $u=\tanh \chi, \operatorname{sech}^{2} \chi=1-u^{2}, \mathrm{~d} u=\left(1-u^{2}\right) \mathrm{d} \chi$, this simplifies to,

$$
\alpha_{1}(u)=\frac{1}{2}\left[\sum_{j} c_{0, j} \sqrt{2 j+1}\left(\int_{u}^{1} P_{j}\left(u^{\prime}\right) \mathrm{d} u^{\prime}-\int_{-1}^{u} P_{j}\left(u^{\prime}\right) \mathrm{d} u^{\prime}\right)\right] .
$$

In terms of the $u$ variable, the modified Vlasov equation (Equation 21) becomes,

$$
\frac{\partial \tilde{f}_{1}}{\partial \tau}+\varpi\left(1-u^{2}\right) \frac{\partial \tilde{f}_{1}}{\partial u}-u \frac{\partial \tilde{f}_{1}}{\partial \varpi}-2 \varpi \alpha_{1}(u) \tilde{f}_{0}=0
$$

Substituting Equation 23 and canceling a common factor of $\tilde{f}_{0}$ produces,

$$
\sum_{i, j}\left\{\dot{c}_{i, j} H_{i} P_{j}+c_{i, j} \varpi H_{i}\left(1-u^{2}\right) \frac{\partial P_{j}}{\partial u}-c_{i, j} u P_{j} \frac{\partial H_{i}}{\partial \varpi}\right\}-2 \varpi \alpha_{1}(u)=0 .
$$

Applying standard Hermite and Legendre polynomial recursion relations to Equation 27 and using the fact that $P_{n}(1)=1$ and $P_{n}(-1)=(-1)^{n}$ in the simplification of $\alpha_{1}$, results in,

$$
\begin{aligned}
& \sum_{i, j} \sqrt{\frac{2 j+1}{2^{i} i !}}\left\{\dot{c}_{i, j} H_{i} P_{j}+c_{i, j}\left[\frac{j(j+1)}{2(2 j+1)} H_{i+1} P_{j-1}-\right.\right. \\
& \frac{j(j+1)}{2(2 j+1)} H_{i+1} P_{j+1}+\frac{i j(j-1)}{2 j+1} H_{i-1, j-1}- \\
& \left.\left.\frac{i(j+1)(j+2)}{2 j+1} H_{i-1} P_{j+1}+\delta_{0, i} \frac{1}{2 j+1} H_{1}\left[P_{j+1}-P_{j-1}\right]\right]\right\}=0 .
\end{aligned}
$$

The term containing the Kroenecker delta corresponds to the $2 \varpi \alpha_{1}(\chi) \tilde{f}_{0}$ term in Equation 21 and is zero except when $i=0$. Finally, we obtain the equations of motion for the coefficients by multiplying this expression by $G_{m, n} \tilde{f}_{0}$, integrating over $\varpi$ and $u$, and making use of the orthogonality relations Equations 16-17. The resulting expressions have the form,

$$
\begin{aligned}
\dot{c}_{m, n}= & L_{m, n}^{m-1, n-1} c_{m-1, n-1}+L_{m, n}^{m-1, n+1} c_{m-1, n+1}+ \\
& L_{m, n}^{m+1, n-1} c_{m+1, n-1}+L_{m, n}^{m+1, n+1} c_{m+1, n+1},
\end{aligned}
$$


where the matrix elements $L_{m, n}^{i, j}$ are given by

$$
\begin{aligned}
L_{m, n}^{m-1, n-1} & =\frac{\sqrt{m}(n-1) n-2 \delta_{1, m}}{\sqrt{2(2 n+1)(2 n-1)}} \\
L_{m, n}^{m-1, n+1} & =-\frac{\sqrt{m}(n+2)(n+1)-2 \delta_{1, m}}{\sqrt{2(2 n+1)(2 n+3)}} \\
L_{m, n}^{m+1, n-1} & =\frac{\sqrt{m+1}(n+1) n}{\sqrt{2(2 n+1)(2 n-1)}} \\
L_{m, n}^{m+1, n+1} & =-\frac{\sqrt{m+1}(n+1) n}{\sqrt{2(2 n+1)(2 n+3)}}
\end{aligned}
$$

where $m, n, i, j \geq 0$. The test-particle case is obtained by omitting the Kronecker $\delta_{1, m}$ terms.

Equations 29-30 are the main results of this paper. For linearized dynamics the $c_{m, n}$ evolve by coupling to diagonal neighbors only. This is somewhat surprising in light of the long-range nature of the forces, and can be traced back to the recursion relation that replaces the integral over $\chi$ in the calculation of $\alpha_{1}$. Because of this nearest-diagonal-neighbor coupling, the even parity and odd parity modes completely decouple, where the parity is given by $(-1)^{m+n}$. For simplicity, we shall concern ourselves with the even parity modes only, and set all the odd parity coefficients to zero. This automatically guarantees that the center of mass velocity and position are zero, $\langle\varpi\rangle=\langle\chi\rangle=0$.

\section{Discussion}

We have demonstrated that a set of orthonormal polynomial terms based on the equilibrium distribution function is useful for investigating the evolution of one-dimensional, self-gravitating, collisionless systems, at least for small linear perturbations from equilibrium. The polynomial coefficients interact via diagonal-neighbor couplings, producing an alternate view of the evolution of these systems in terms of coefficients $c_{m, n}$ on the $(m, n)$ grid.

This polynomial expansion analysis of the Vlasov equation provides a novel, and useful, view of the behavior of one-dimensional self-gravitating systems. While not in the scope of this introductory work, one can imagine several directions any future investigations using this analysis might take. For example one might study the aftermath of collisions of isolated systems, or the stationary states of one-dimensional systems, or the frequency spectrum of the $L$ matrix. One could extend the analysis to second-order to investigate the onset of nonlinear effects, like stability or chaotic behavior. The nearest-neighbor coupling of the coefficients leads to "local" continuity-type dynamics of conserved quantities like energy and fine-grained entropy on the $(m, n)$ grid that should give further insight into the nonequilibrium thermodynamics of these systems. 


\section{REFERENCES}

Alvord J., Miller B.N., 2009, in Joint Fall 2009 Meeting of the Texas Sections of the APS, AAPT, and SPS. American Phys. Soc., p. C5.002

Binney J., Tremaine S., 1987, Galactic Dynamics. Princeton Univ. Press, Princeton, NJ

Camm G.L., 1950, MNRAS, 110, 305

Clowe D., Bradac M., Gonzalez A.H., Markevitch M., Randall S.W., Jones C., Zaritsky D., 2006, ApJ, 648, L109

Hansen, S.H., Moore B., 2006, New Ast., 11, 333

Joyce, M., Worrakitpoonpon, T., 2011, Phys. Rev. E, 84, 1139

Koyama, H., Konishi T., 2001, Phys. Lett. A, 279, 226

Landau L.D., Lifshitz E.M., 1951, Statistical Physics - Part 1. Butterworth-Heinemann, Boston, MA

Lithwick Y., Dalal N, 2011, ApJ, 734, 100

Merritt D., Aguilar L.A., 1985, MNRAS, 217, 787

Mohr J.J., Evrard A.E., Fabricant D.G., Geller M.J., 1995, ApJ, 447, 8

Navarro J.F., Frenk C.S., White S.D.M., 1996, ApJ, 462, 563

Navarro J.F., Frenk C.S., White S.D.M., 1997, ApJ, 490, 493

Navarro J.F. et al., 2004, MNRAS, 349, 1039

Noullez A., Aurell E., Fanelli D., 2003, J. Comp. Phys., 186, 697

Reidl, Jr., C.J., Miller B.N., 1987, ApJ, 318, 248

Reidl, Jr., C.J., Miller B.N., 1988, ApJ, 332, 619

Romanowsky A.J., Douglas N.G., Arnaboldi M., Kuijken K., Merrifield M.R., Napolitano N.R., Capaccioli M., Freeman K.C., 2003, Science, 301, 1696

Rubin, V.C, Ford W.K., 1970, ApJ, 159, 379

Schulz A.E., Dehnen W., Jungman G., Tremaine S., 2013, MNRAS, 431, 49

Spergel D.N. et al., 2003, ApJS, 148, 175

Springel V. et al., 2005, Nature, 435, 629

Taylor J., Navarro J., 2001, ApJ, 563, 483

Valageas P., 2006, A\&A, 450, 445

Williams L.L.R, Saha P., 2011, MNRAS, 415, 448

Zwicky F., 1937, ApJ, 86, 217 